\title{
Paper
}

\section{Performance Evaluation of Elliptical and Multi-part Mirrors for Concentrating Diffused Light onto Collector Surface from Half Celestial Sphere}

\author{
Mitsuhiro Matsumoto* ${ }^{* \dagger}$ Member
}

(Received February 3, 2021, revised July 19, 2021)

\begin{abstract}
An elliptical mirror has a mirror surface at the first focal point on a plane perpendicular to its central axis, which, in turn, passes through the first and second focal points. It can collect diffused light from a half celestial sphere, which extends $360^{\circ}$ in the axial rotation direction and $180^{\circ}$ in the axial direction at the second focal point. An elliptical mirror allows only the light incident on the first focal point to be concentrated on only the second focal point. A single elliptical mirror consists of only one elliptical mirror. A multi-part mirror is composed of multiple elliptical mirrors. In a multi-part elliptical mirror, the first focal point lies on its surface, and the second focal point is so positioned that it is aligned with the top of its surface. A multi-part mirror can concentrate diffused light from a wider area than a single elliptical mirror because its first focal points are dispersed. Furthermore, in a single elliptical mirror, only the light incident on the first focal point is concentrated on only the second focal point; therefore, the light-concentrating efficiency is low. In this study, we installed a collector surface on the second focal point of an elliptical mirror to increase its light-concentrating efficiency. Subsequently, we evaluated single elliptical and multi-part mirrors that capture the diffused light from a half celestial sphere not incident on the first focal point and concentrate it on the collector surface as well as the second focal point. We configured a coordinate system for the design of single elliptical and multi-part mirrors and subsequently analyzed the mechanism by which the light will strike these designed mirrors and concentrate on their collector surfaces. Based on the design values, we manufactured actual single elliptical and multi-part mirrors, which were illuminated with diffused light, and measured the illuminance of the light concentrated on their collector surfaces. Finally, we evaluated the light-concentrating performance of these mirrors based on the analysis results and measured illumination.
\end{abstract}

Keywords: Elliptical Mirror, Multi-part Mirror, Light Concentrating, Diffused Light, Collector Surface, Half Celestial Sphere, Illuminance, Illuminance Ratio

\section{Introduction}

Light-concentrating collectors, which employ a concentrator to concentrate direct sunlight and convert this concentrated light energy into heat energy by emitting it onto a heat collector at the collector surface are widely used [1]. Plane mirrors, concave mirrors, composite parabolic mirrors, and Fresnel lenses are used as concentrators [1]. However, a light-concentrating collector only concentrates direct light from the sun and cannot concentrate diffused light from the entire sky, which is a half celestial sphere [2]. The proportion of diffused light is high in most parts of the world, except in desert areas where the weather is sunny all year round [2]. In Japan, approximately half of the total annual solar radiation is diffused light [2]. In particular, on the Sea of Japan side from San'in to Hokkaido, there is an average cloud cover of 8.5 or more for more than 180 days during

\footnotetext{
* Corresponding: m-matsumoto@kanagawa-u.ac.jp

$\dagger$ Faculty of Engineering, Kanagawa University, 3-27-1, Rokkakubashi, Kanagawa-ku, Yokohama-shi, Kanagawa, 221-8686, Japan
}

which only diffused light irradiates [3]. Therefore, locations such as the Sea of Japan side of Japan require a concentrator that can concentrate the diffused light from a half celestial sphere on the collector surface.

A variety of research has been conducted on concentrators for concentrating diffused light. Winston, Derrick et al., and Rao et al. presented composite parabolic mirrors arranged in parallel [4] [6]. Mather et al. and Mills et al. presented symmetric and asymmetric cylindrical mirrors [7] [8]. Mather et al. and Derrick et al. proposed an involute-shaped mirror [5] [7]. Bassett et al. suggested mirrors with symmetrical and asymmetric shapes [9]. Grimmer, Frissora et al., Norton et al., Suzuki, Eames et al., and Wang et al. presented composite parabolic mirrors possessing symmetric and asymmetric shapes [10] [16]. Grimmer and Derrick et al. presented a parabolic mirror [5] [10]. Derrick et al. recommended a plane mirror [5]. Yamada et al. proposed Fresnel lenses, cylindrical lenses, water cylinder lenses, and parabolic mirrors [17]. However, all these studies suggested two-dimensional concentrators, which are 
Table 1: List of variables used in equations.

\begin{tabular}{|c|c|c|c|}
\hline Variable & Meaning & Variable & Meaning \\
\hline$a$ & Variable in Eq. (15) & $r_{\mathrm{F} j 0}$ & Coordinate of $\mathrm{F}_{j 0}$ \\
\hline$b$ & Variable in Eq. (15) & $r_{\mathrm{F} 00}$ & Coordinate of $\mathrm{F}_{00}$ \\
\hline$e$ & Illuminance ratio & $r_{j k}$ & Coordinate in $\mathrm{R}_{j k}-\Theta_{j k}-\mathrm{Z}_{j k}$ \\
\hline$f$ & Focal length of the ellipse & $r_{\mathrm{R} m}$ & Coordinate of $\mathrm{R}_{m}$ \\
\hline$\phi_{\mathrm{N} m}$ & Angle between normal of ellipse at $\mathrm{R}_{m}$ and $\mathrm{R}_{j k}$ & $r_{\mathrm{R} m-1}$ & Coordinate of $\mathbf{R}_{m-1}$ \\
\hline$\phi_{\mathrm{R} m}$ & Angle between reflected light at $\mathrm{R}_{m}$ and $\mathrm{R}_{j k}$ & $r_{\mathrm{R} 0}$ & Coordinate of $\mathrm{R}_{0}$ \\
\hline$\phi_{\mathrm{R} m-1}$ & Angle between reflected light at $\mathrm{R}_{m-1}$ and $\mathrm{R}_{j k}$ & $r_{00}$ & Coordinate in $\mathrm{R}_{00}-\Theta_{00}-\mathrm{Z}_{00}$ \\
\hline$\phi_{\mathrm{R} 0}$ & Angle between incident light at $\mathrm{R}_{0}$ and $\mathrm{R}_{j k}$ & $\theta_{\mathrm{F} j}$ & Angle from $\mathrm{F}_{j 0}$ to $\mathrm{F}_{j 1}$ \\
\hline$g$ & Distance from edge to another edge of mirror surface & $\theta_{\mathrm{F} j k}$ & Coordinate of $\mathrm{F}_{j k}$ \\
\hline$h$ & Height of the elliptical mirror & $\theta_{\mathrm{F} j 0}$ & Coordinate of $\mathrm{F}_{j 0}$ \\
\hline$i_{\mathrm{D}}$ & Illuminance of the dome light & $\theta_{\mathrm{F} 00}$ & Coordinate of $\mathrm{F}_{00}$ \\
\hline$i_{\mathrm{M}}$ & Illuminance of the measured object & $\xi_{\mathrm{F}}$ & Angle from $\mathrm{F}_{00}$ to $\mathrm{F}_{10}$ \\
\hline$i_{\mathrm{S}}$ & Illuminance of the light-shielded measurement object & $\xi_{\mathrm{F} j}$ & Angle from $\mathrm{R}_{00}-\Theta_{00}-\mathrm{Z}_{00}$ to $\mathrm{R}_{j 0}-\Theta_{j 0}-\mathrm{Z}_{j 0}$ \\
\hline$i_{\mathrm{T}}$ & Illuminance of the only measured object & $z$ & Coordinate in $\mathrm{R}-\Theta-\mathrm{Z}$ \\
\hline$j$ & Number indicating elliptical mirror in the R-axis & $z_{\mathrm{F} j k}$ & Coordinate of $\mathrm{F}_{j k}$ \\
\hline$k$ & Number indicating elliptical mirror in the $\Theta$-axis & $z_{\mathrm{F} j 0}$ & Coordinate of $\mathrm{F}_{j 0}$ \\
\hline$m$ & Number of reflections & $z_{\mathrm{F} 00}$ & Coordinate of $\mathrm{F}_{00}$ \\
\hline$n_{\mathrm{M}}$ & Number of all elliptical mirrors & $z_{j k}$ & Coordinate in $\mathrm{R}_{j k}-\Theta_{j k}-\mathrm{Z}_{j k}$ \\
\hline$n_{\mathrm{R}}$ & Number of elliptical mirrors in the R-axis & $z_{\mathrm{R} m}$ & Coordinate of $\mathrm{R}_{m}$ \\
\hline$n_{\Theta j}$ & Number of elliptical mirrors in the $\Theta$-axis & $z_{\mathrm{R} m-1}$ & Coordinate of $\mathrm{R}_{m-1}$ \\
\hline$r$ & Coordinate in R- $\Theta-\mathrm{Z}$ & $z_{00}$ & Coordinate in $\mathrm{R}_{00}-\Theta_{00}-\mathrm{Z}_{00}$ \\
\hline$r_{\mathrm{C}}$ & Coordinate of $\mathrm{C}$ & $2 l$ & Length of the major axis of ellipse \\
\hline$r_{\mathrm{E}}$ & Radius of the elliptical mirror & $2 s$ & Length of the minor axis of ellipse \\
\hline$r_{\mathrm{F} j k}$ & Coordinate of $\mathrm{F}_{j k}$ & - & - \\
\hline
\end{tabular}

inadequate for concentrating the diffused light from a threedimensional half celestial sphere. Other studies have been conducted on three-dimensional concentrators. Sellami et al. presented a square elliptical hyperbolic mirror [18]. However, it emphasized the injection of direct light into a solar cell.

Matsumoto presented a multi-part mirror arranged with an array of elliptical mirrors [19]. An elliptical mirror has a mirror surface at the first focal point on a plane perpendicular to its central axis, which, in turn, passes through the first and second focal points. It can collect diffused light from a half celestial sphere that extends $360^{\circ}$ in the axial rotation direction and $180^{\circ}$ in the axial direction at the second focal point. An elliptical mirror allows only the light incident on the first focal point to be concentrated on only the second focal point. A multi-part mirror is composed of multiple elliptical mirrors. In all the elliptical mirrors of a multi-part mirror, the first focal point is located on the surface of the multi-part mirror, and the second focal point is positioned so that it is aligned to its top. A multi-part mirror can concentrate diffused light from a wider area than a single elliptical mirror because the first focal points of the former are dispersed on its surface. However, in a multipart mirror, only the light incident on the first focal point is concentrated on only the second focal point; therefore, the light-concentrating efficiency is low.

In this study, we installed a collector surface on the second focal point of an elliptical mirror, to increase its lightconcentrating efficiency. Subsequently, we evaluated single elliptical and multi-part mirrors that capture the diffused light from a half celestial sphere not incident on the first focal point and concentrate it on the collector surface as well as the second focal point. We configured a coordinate system for the design of single elliptical mirrors and multipart mirrors and subsequently analyzed the mechanism by which the light is incident on our designed single elliptical and multi-part mirrors and concentrates on their collector surfaces. Based on the design values, we manufactured actual single elliptical and multi-part mirrors. Using our experimental set-up, we illuminated the manufactured mirrors with diffused light and measured the illuminance of the light concentrated on their collector surfaces. Finally, we evaluated the light-concentrating performance of these single elliptical and multi-part mirrors based on the analysis results and the measured illumination.

\section{Design}

2.1 Elliptical Mirror Table 1 lists the variables in the equations used in this study. We set up the coordinate system of an elliptical mirror to design single elliptical mirrors and elliptical mirrors for multi-part mirrors. Figure 1 presents the elliptical mirror coordinate system and design method. Figures 1(a) and (b) show the front and longitudinal views, respectively. In the coordinate system, $\mathrm{F}_{j k}$, and the origin, $\mathrm{O}_{j k}$, are the first and second focal points of the elliptical mirror, respectively. The central axis of the elliptical mirror passes through the first focal point, $\mathrm{F}_{j k}$, and the origin, $\mathrm{O}_{j k}$. The coordinate system of the elliptical mirror is $\mathrm{R}_{j k}-\Theta_{j k}-\mathrm{Z}_{j k}$, where the $\mathrm{R}_{j k}$ axis is the radial direction, $\Theta_{j k}$ axis is the direction of rotation around the center axis, and $\mathrm{Z}_{j k}$ axis is the center axis direction. The elliptical mirrors are indexed as $j$ and $k$. Coordinates in the $\mathrm{R}_{j k}-\Theta_{j k}-\mathrm{Z}_{j k}$ co- 


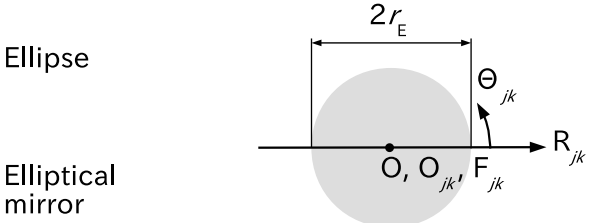

(a) Front view

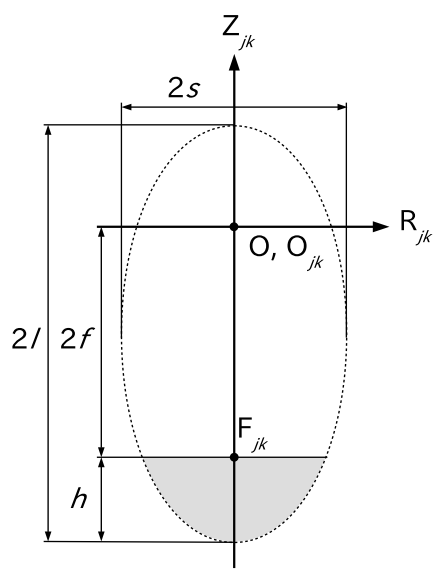

(b) Longitudinal view

Figure 1: Coordinate system and design method of elliptical mirror at front view and longitudinal view.

$$
\begin{aligned}
& \text { Elliptical } \\
& \text { mirror } \\
& \text { - } \mathrm{F}_{00} \quad\left(r_{\mathrm{FOO}}, \theta_{\mathrm{FOO}}, z_{\mathrm{FOO}}\right) \\
& \text { - } F_{10} \quad\left(r_{\mathrm{F}_{10}}, \theta_{\mathrm{F} 10^{\prime}} z_{\mathrm{F} 10^{\prime}}\right) \\
& \text { - } \mathrm{F}_{j 0} \quad\left(r_{\mathrm{F} j 0^{\prime}}, \theta_{\mathrm{Fj} 0^{\prime}}, z_{\mathrm{Fjo}}\right) \\
& \text { - } F_{j k} \quad\left(r_{F j k^{\prime}} \theta_{F_{j k},} z_{F j k}\right) \\
& \hookrightarrow \xi_{\mathrm{F}} / 2 \\
& \longleftrightarrow 2 f \\
& \leftrightarrow r_{\mathrm{E}} \\
& \longleftrightarrow g / 2 \\
& \longleftrightarrow \xi_{\mathrm{F} j} \\
& \longleftrightarrow \theta_{\mathrm{Fj}} / 2 \\
& \longleftrightarrow r_{\mathrm{Fjo}} \\
& \longleftrightarrow \theta_{F j k}
\end{aligned}
$$

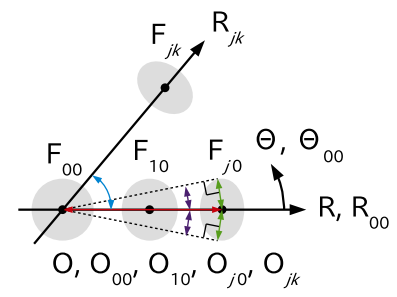

(a) Front view

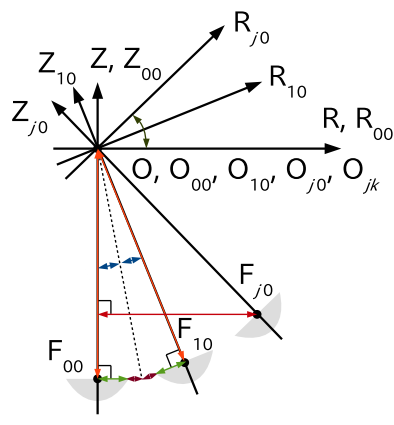

(b) Longitudinal view

Figure 2: Coordinate system and design method of single elliptical and multi-part mirrors at front view and longitudinal view.

ordinate system are denoted as $\left(r_{j k}, \theta_{j k}, z_{j k}\right)$.

An elliptical mirror has a three-dimensional shape obtained by rotating an ellipse around the $Z_{j k}$ axis on the $R_{j k}-$
$\mathrm{Z}_{j k}$ plane in the $\mathrm{R}_{j k}-\Theta_{j k}-\mathrm{Z}_{j k}$ coordinate system, which allows axisymmetry to be achieved with respect to the $Z_{j k}$ axis. The focal length, length of the major axis, and length of the minor axis of the ellipse are denoted as $f, 2 l$, and $2 s$, respectively. The mirror surface is at the first focal point, $\mathrm{F}_{j k}$, on a plane perpendicular to the $Z_{j k}$ axis and parallel to the $\mathrm{R}_{j k}-\Theta_{j k}$ plane in the $\mathrm{R}_{j k}-\Theta_{j k}-Z_{j k}$ coordinate system passing through the first focal point, $\mathrm{F}_{j k}$. The radius and height of the elliptical mirror are denoted as $r_{\mathrm{E}}$ and $h$, respectively.

The range of the light incident on the elliptical mirror is a half celestial sphere from the first focal point, $\mathrm{F}_{j k}$, in the direction of the origin, $\mathrm{O}_{j k}$. In the design of the elliptical mirror, focal length $f$, radius $r_{\mathrm{E}}$, and height $h$ are determined using the equations below. The focal length, $f$, can be expressed as

$$
f=\sqrt{l^{2}-s^{2}} \quad(l>s>0)
$$

Using Eq. (1), the equation of the ellipse can be expressed as

$$
\frac{r_{j k}^{2}}{s^{2}}+\frac{\left(z_{j k}+2 f\right)^{2}}{l^{2}}=1 \quad\left(z_{j k} \leqq-2 f\right)
$$

Using Eq. (2), the radius, $r_{\mathrm{E}}$, can be expressed as

$$
r_{\mathrm{E}}=\frac{s^{2}}{l}
$$

Using Eq. (1), the height, $h$, can be expressed as

$$
h=l-f
$$

\subsection{Single Elliptical Mirror and Multi-part Mirrors} We set up a coordinate system for designing single elliptical and multi-part mirrors. Figure 2 shows the coordinate system and design method for these single elliptical and multipart mirrors. Figures 2(a) and (b) present the front and longitudinal views, respectively. A single elliptical mirror has a first focal point $\mathrm{F}_{00}$. A multi-part mirror is composed of multiple elliptical mirrors with multiple first focal points, $\mathrm{F}_{j k}$. For each elliptical mirror of a multi-part mirror, the first focal point, $\mathrm{F}_{j k}$, lies on the surface of the multi-part mirror. The elliptical mirrors are positioned on the surface of the multi-part mirror. Adjacent elliptical mirrors are arranged with a certain distance between them. The origin, $\mathrm{O}_{j k}$, which is the second focal point of all the elliptical mirrors, is aligned with the top of the multi-part mirror surface.

In the coordinate system, the origin, $\mathrm{O}$, denotes the second focal point of all the elliptical mirrors, and the central axis of a single elliptical or multi-part mirror passes through this point. The coordinate system is defined as R$\Theta-Z$, where $R$ axis is the radial direction, $\Theta$ axis is the direction of rotation around the center axis, and $\mathrm{Z}$ axis is the center axis direction. Coordinates in the $\mathrm{R}-\Theta-\mathrm{Z}$ coordinate system are denoted as $(r, \theta, z)$. For an elliptical mirror with a first focal point $\mathrm{F}_{00}$ and origin $\mathrm{O}_{00}$, the $\mathrm{R}_{00}, \Theta_{00}$, and $\mathrm{Z}_{00}$ axes in the $R_{00}-\Theta_{00}-Z_{00}$ coordinate system coincide with the origin, $O$, and the $R, \Theta$, and $Z$ axes in the $R-\Theta-Z$ coordinate system. Coordinates in the $R_{00}-\Theta_{00}-Z_{00}$ coordinate system are denoted as $\left(r_{\mathrm{F} 00}, \theta_{\mathrm{F} 00}, z_{\mathrm{F} 00}\right)$. 
For an elliptical mirror having a first focal point $\mathrm{F}_{j 0}$, the $\mathrm{R}_{j 0}-\Theta_{j 0}-\mathrm{Z}_{j 0}$ coordinate system is the coordinate system obtained by rotating the $R_{00}-\Theta_{00}-Z_{00}$ coordinate system at an angle of $\xi_{\mathrm{F} j}$ about the origin, $\mathrm{O}_{00}$, on the $\mathrm{R}_{00}-\mathrm{Z}_{00}$ plane. The index, $j$, denotes the number of elliptical mirrors rotated accordingly, where $j$ is a natural number including 0 . In this rotation, $g$ denotes the edge-to-edge distance on the mirror surface from the first focal point, $\mathrm{F}_{00}$, to first the focal point of an adjacent elliptical mirror, $\mathrm{F}_{10}$, and $\xi_{\mathrm{F}}$ denotes the corresponding angle. Angle $\xi_{\mathrm{F}}$ is found using trigonometry based on the geometry of angle $\xi_{\mathrm{F}}$, focal length $f$, radius $r_{\mathrm{E}}$, and distance $g$, which are shown in Fig. 2(b). For this rotation, the number of elliptical mirrors is denoted as $n_{\mathrm{R}}$. Coordinates in the $\mathrm{R}_{j 0}-\Theta_{j 0}-Z_{j 0}$ coordinate system are denoted as $\left(r_{\mathrm{F} j 0}, \theta_{\mathrm{F} j 0}, z_{\mathrm{F} j 0}\right)$.

For an elliptical mirror with a first focal point $\mathrm{F}_{j k}$, the $\mathrm{R}_{j k^{-}}$ $\Theta_{j k}-Z_{j k}$ coordinate system is defined as a coordinate system rotated at an angle $\theta_{\mathrm{F} j k}$ about the $\mathrm{Z}_{00}$ axis centered in the $\mathrm{R}_{j 0}-\Theta_{j 0}-\mathrm{Z}_{j 0}$ coordinate system. Index $k$ denotes the number of elliptical mirrors rotated accordingly, where $k$ is a natural number including 0 . In this rotation, $\theta_{\mathrm{F} j}$ denotes the angle from the first focal point, $\mathrm{F}_{j 0}$, to the first focal point of an adjacent elliptical mirror, $\mathrm{F}_{j 1}$. Angle $\theta_{\mathrm{F} j}$ is found using trigonometry based on the geometry of angle $\theta_{\mathrm{F} j}$, radius $r_{\mathrm{E}}$, and radius $r_{\mathrm{F} j 0}$, which are presented in Fig. 2(a). For this rotation, the number of elliptical mirrors is denoted as $n_{\Theta j}$. The number, $n_{\Theta j}$, is a natural number rounded off to the nearest whole number. Coordinates in the $\mathrm{R}_{j k}-\Theta_{j k}-\mathrm{Z}_{j k}$ coordinate system are denoted as $\left(r_{\mathrm{F} j k}, \theta_{\mathrm{F} j k}, z_{\mathrm{F} j k}\right)$.

The total number of elliptical mirrors is denoted as $n_{\mathrm{M}}$. Note that when $j$ and $k$ are 0 , it is a single elliptical mirror. A multi-part mirror disperses the first focal points over its surface more widely than a single elliptical mirror. Using a multi-part mirror, it is possible to concentrate diffused light from a wider area than using a single elliptical mirror. In the designs of the single elliptical and multi-part mirrors, the number of mirrors $n_{\mathrm{M}}$, angle $\xi_{\mathrm{F} j}$, number $n_{\Theta j}$, angle $\theta_{\mathrm{F} j}$, coordinate $r_{\mathrm{F} j k}$, and coordinate $z_{\mathrm{F} j k}$ are determined using the equations below. Using Eq. (1), coordinates $\left(r_{\mathrm{F} 00}, \theta_{\mathrm{F} 00}\right.$, $\left.z_{\mathrm{F} 00}\right)$ can be expressed as

$$
\left(\begin{array}{c}
r_{\mathrm{F} 00} \\
\theta_{\mathrm{F} 00} \\
z_{\mathrm{F} 00}
\end{array}\right)=\left(\begin{array}{c}
0 \\
0 \\
-2 f
\end{array}\right)
$$

Using Eqs. (1) and (3), the relationship between angle $\xi_{\mathrm{F}}$, focal length $f$, radius $r_{\mathrm{E}}$, and distance $g$ can be expressed as

$$
\tan \frac{\xi_{\mathrm{F}}}{2}=\frac{r_{\mathrm{E}}+\frac{g}{2}}{2 f}
$$

Using Eq. (6), angle $\xi_{\mathrm{F} j}$ can be expressed as

$$
\xi_{\mathrm{F} j}=j \xi_{\mathrm{F}} \quad\left(j=0,1, \cdots, n_{\mathrm{R}}-1\right)
$$

Using Eqs. (5) and (7), coordinates $\left(r_{\mathrm{F} j 0}, \theta_{\mathrm{F} j 0}, z_{\mathrm{F} j 0}\right)$ can be expressed as

$$
\left(\begin{array}{c}
r_{\mathrm{F} j 0} \\
\theta_{\mathrm{F} j 0} \\
z_{\mathrm{F} j 0}
\end{array}\right)=\left(\begin{array}{ccc}
\cos \xi_{\mathrm{F} j} & 0 & -\sin \xi_{\mathrm{F} j} \\
0 & 1 & 0 \\
\sin \xi_{\mathrm{F} j} & 0 & \cos \xi_{\mathrm{F} j}
\end{array}\right)\left(\begin{array}{c}
r_{\mathrm{F} 00} \\
\theta_{\mathrm{F} 00} \\
z_{\mathrm{F} 00}
\end{array}\right)
$$

Using Eqs. (3) and (8), the relationship between angle $\theta_{\mathrm{F} j}$, radius $r_{\mathrm{E}}$, and radius $r_{\mathrm{F} j 0}$ can be expressed as

$$
\sin \frac{\theta_{\mathrm{F} j}}{2}=\frac{r_{\mathrm{E}}}{r_{\mathrm{F} j 0}}
$$

Using Eq. (9), number $n_{\Theta j}$ can be expressed as

$$
n_{\Theta j}=\left[\frac{2 \pi}{\theta_{\mathrm{F} j}}\right]
$$

Using Eq. (10), angle $\theta_{\mathrm{F} j}$ can be expressed as

$$
\theta_{\mathrm{F} j}=\frac{2 \pi}{n_{\Theta j}}
$$

Using Eqs. (10) and (11), angle $\theta_{\mathrm{F} j k}$ can be expressed as

$$
\theta_{\mathrm{F} j k}=k \theta_{\mathrm{F} j} \quad\left(k=0,1, \cdots, n_{\Theta j}-1\right)
$$

Using Eqs. (8) and (12), coordinates $\left(r_{\mathrm{F} j k}, \theta_{\mathrm{F} j k}, z_{\mathrm{F} j k}\right)$ can be expressed as

$$
\left(\begin{array}{c}
r_{\mathrm{F} j k} \\
\theta_{\mathrm{F} j k} \\
z_{\mathrm{F} j k}
\end{array}\right)=\left(\begin{array}{c}
0 \\
\theta_{\mathrm{F} j k} \\
0
\end{array}\right)+\left(\begin{array}{c}
r_{\mathrm{F} j 0} \\
\theta_{\mathrm{F} j 0} \\
z_{\mathrm{F} j 0}
\end{array}\right)
$$

Using Eq. (10), the number of mirrors, $n_{\mathrm{M}}$, can be expressed as

$$
n_{\mathrm{M}}=\sum_{j=0}^{n_{\mathrm{R}}-1} n_{\Theta j}
$$

To evaluate the light-concentrating performance of a single elliptical mirror, we designed three types of it with different focal lengths $f$ and radii $r_{\mathrm{E}}$. Table 2 lists the design values for these single elliptical mirrors. The design values of the single elliptical mirror with a focal length $f$ of $199.929 \mathrm{~mm}$ and radius $r_{\mathrm{E}}$ of $1 \mathrm{~mm}$ are based on the values provided by Matsumoto [19]. To evaluate the lightconcentrating performance of the multi-part mirrors, we designed two types with different focal lengths $f$. The design values of the multi-part mirrors are summarized in Tables 3 and 4 . Table 3 and 4 list the data for multi-part mirrors with focal lengths $f$ of $17.503 \mathrm{~mm}$ and $199.929 \mathrm{~mm}$, respectively. The design values in Table 4 are based on the values obtained by Matsumoto [19]. In Tables 2-4, the design values are determined using Eqs. (1), (3), (4), (14), (7), (10), (11), and (13) for focal length $f$, radius $r_{\mathrm{E}}$, height $h$, number of mirrors $n_{\mathrm{M}}$, angle $\xi_{\mathrm{F} j}$, number $n_{\Theta j}$, angle $\theta_{\mathrm{F} j}$, and coordinates $r_{\mathrm{F} j k}$ and $z_{\mathrm{F} j k}$, respectively.

\section{Analysis Method}

In single elliptical and multi-part mirrors, the diffused light incident on the elliptical mirrors is reflected by the mirrored surface and concentrated on the collector surface at the second focal point. To evaluate the light-concentrating performance of the designed single elliptical and multi-part mirrors, we analyzed how much of the light incident on them was concentrated on their collector surface. Figure 3 shows the analysis method for these single elliptical and multi-part 
Table 2: Design values of length of major axis, length of minor axis, focal length, radius of elliptical mirror, height, number of elliptical mirrors in R-axis, number of all elliptical mirrors, angle from $R_{00}-\Theta_{00}-Z_{00}$ to $R_{00}-\Theta_{00}-Z_{00}$, number of elliptical mirrors in $\Theta$-axis, angle from $F_{00}$ to $F_{01}$, and coordinate of $F_{00}$ in single elliptical mirrors.

\begin{tabular}{r|r|r|r|r|r|r|r|r|r|r|r}
$l[\mathrm{~mm}]$ & $s[\mathrm{~mm}]$ & $f[\mathrm{~mm}]$ & $r_{\mathrm{E}}[\mathrm{mm}]$ & $h[\mathrm{~mm}]$ & $n_{\mathrm{R}}$ & $n_{\mathrm{M}}$ & $\xi_{\mathrm{F} 0}\left[^{\circ}\right]$ & $n_{\Theta 0}$ & $\theta_{\mathrm{F} 0}\left[^{\circ}\right]$ & $r_{\mathrm{F} 00}[\mathrm{~mm}]$ & $z_{\mathrm{F} 00}[\mathrm{~mm}]$ \\
\hline \hline 18.000 & 4.200 & 17.503 & 0.980 & 0.497 & 1 & 1 & 0.000 & 1 & 0.000 & 0.000 & -35.006 \\
\hline 40.300 & 36.300 & 17.504 & 32.697 & 22.796 & 1 & 1 & 0.000 & 1 & 0.000 & 0.000 & -35.008 \\
\hline 200.000 & 14.100 & 199.929 & 1.000 & 0.498 & 1 & 1 & 0.000 & 1 & 0.000 & 0.000 & -399.858
\end{tabular}

Table 3: Design values of length of major axis, length of minor axis, focal length, radius of elliptical mirror, height, distance from one edge to another edge of mirror surface, number of elliptical mirrors in R-axis, number of all elliptical mirrors, number indicating elliptical mirror in R-axis, angle from $R_{00}-\Theta_{00}-Z_{00}$ to $R_{j 0}-\Theta_{j 0}-Z_{j 0}$, number of elliptical mirrors in $\Theta$-axis, angle from $F_{j 0}$ to $F_{j 1}$, and coordinates of $F_{j k}$ in multi-part mirror when $f$ is $17.503 \mathrm{~mm}$.

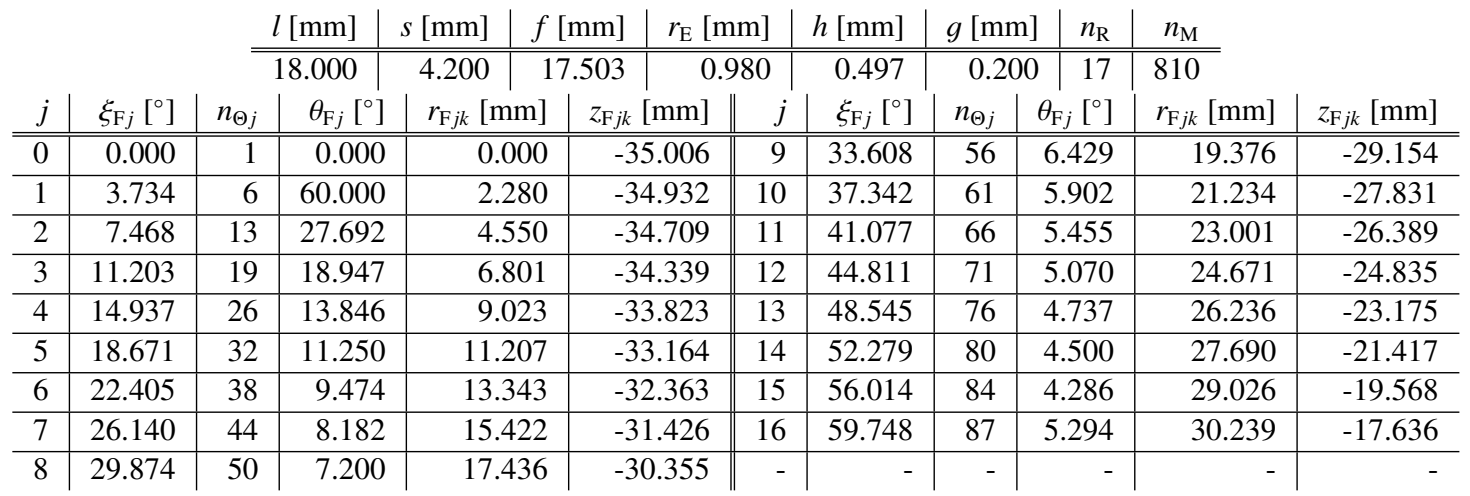

mirrors. An elliptical mirror is three-dimensional in shape, which is obtained by rotating an ellipse around the $\mathrm{Z}_{j k}$ axis on the $\mathrm{R}_{j k}-\mathrm{Z}_{j k}$ plane of the $\mathrm{R}_{j k}-\Theta_{j k}-\mathrm{Z}_{j k}$ coordinate system. Therefore, to achieve axisymmetry with respect to the $Z_{j k}$ axis, each elliptical mirror is analyzed in the $\mathrm{R}_{j k}-Z_{j k}$ plane in the $\mathrm{R}_{j k}-\Theta_{j k}-\mathrm{Z}_{j k}$ coordinate system. We analyzed the designed single elliptical and multi-part mirrors in the R-Z plane in the $\mathrm{R}-\Theta-Z$ coordinate system.

The number of reflections of the light in the mirror surface is denoted as $m$, where $m$ is a natural number including 0 . When $m$ is 0 , it is assumed that the light enters the elliptical mirror. This occurs from a line perpendicular to the $\mathrm{Z}_{j k}$ axis that passes through the first focal point, $\mathrm{F}_{j k}$, and is parallel to the $\mathrm{R}_{j k}$ axis. The point of incidence $\mathrm{R}_{0}$ is defined as the point where the parallel line intersects with the incident light. The coordinates of the point of incidence, $\mathrm{R}_{0}$, are $\left(r_{\mathrm{R} 0},-2 f\right)$. Note that the absolute value of the coordinate, $r_{\mathrm{R} 0}$, is less than or equal to the radius, $r_{\mathrm{E}}$. The angle between the point of incidence of the light, $\mathrm{R}_{0}$, and the $\mathbf{R}_{j k}$ axis is denoted as $\phi_{\mathrm{R} 0}$. The reflection points on the mirror surface are denoted as $\mathrm{R}_{m-1}$ and $\mathrm{R}_{m}$. The coordinates of reflection point $\mathbf{R}_{m-1}$ are $\left(r_{\mathrm{R} m-1}, z_{\mathrm{R} m-1}\right)$. The angle between the normal of the ellipse and the $\mathrm{R}_{j k}$ axis at the point of reflection, $\mathbf{R}_{m-1}$, is denoted as $\phi_{\mathrm{N} m-1}$. The angle between the light reflected at the point of reflection, $\mathbf{R}_{m-1}$, and the $\mathbf{R}_{j k}$ axis is denoted as $\phi_{\mathrm{R} m-1}$. The coordinates of the reflection point $\mathrm{R}_{m}$ are $\left(r_{\mathrm{R} m}, z_{\mathrm{R} m}\right)$. The angle between the normal of the ellipse and the $\mathrm{R}_{j k}$ axis at the point of reflection, $\mathrm{R}_{m}$, is denoted as $\phi_{\mathrm{N} m}$. The angle between the light reflected at the point of reflection, $\mathbf{R}_{m}$, and the $\mathbf{R}_{j k}$ axis is denoted as $\phi_{\mathrm{R} m}$.
The collector surface is a line on the $\mathrm{R}$ axis, perpendicular to the $\mathrm{Z}$ axis, and passing through the origin, $\mathrm{O}$, which is the second focal point. The R-Z plane is a plane obtained by rotating the $\mathrm{R}_{00}-\mathrm{Z}_{00}$ plane about the origin $\mathrm{O}_{00}$ by an angle $\xi_{\mathrm{F} j}$. The point of concentration, $\mathrm{C}$, is defined as the point of intersection of the light rays on the collector surface at coordinates $\left(r_{\mathrm{C}}, 0\right)$.

In the analysis of the designed single elliptical and multipart mirrors, the relationship between the angle, $\phi_{\mathrm{R} 0}$, of the coordinate, $r_{\mathrm{R} 0}$, of the light incident on the elliptical mirrors and the coordinate, $r_{\mathrm{C}}$, of the surface where the incoming light collects is found using the equations below. The equation describing the light passing through the point of reflection $\mathrm{R}_{m-1}$ in the $\mathrm{R}_{j k}-\mathrm{Z}_{j k}$ plane can be expressed as follows, where $a$ and $b$ are variables.

$$
z_{j k}=a r_{j k}+b
$$

Variable $a$ can be expressed as

$$
a=\tan \phi_{\mathrm{R} m-1}
$$

Variable $b$ can be expressed as

$$
b=z_{\mathrm{R} m-1}-\tan \phi_{\mathrm{R} m-1} r_{\mathrm{R} m-1}
$$

Using Eqs. (2) and (15), coordinate $r_{\mathrm{R} m}$ can be expressed as

$$
\begin{aligned}
r_{\mathrm{R} m} & =\frac{-a s^{2}(b+2 f)}{l^{2}+a^{2} s^{2}} \\
& +\frac{s \sqrt{a^{2} s^{2}(b+2 f)^{2}-\left(l^{2}+a^{2} s^{2}\right)\left\{(b+2 f)^{2}-l^{2}\right\}}}{l^{2}+a^{2} s^{2}}
\end{aligned}
$$


Table 4: Design values of length of major axis, length of minor axis, focal length, radius of elliptical mirror, height, distance from one edge to another edge of mirror surface, number of elliptical mirrors in R-axis, number of all elliptical mirrors, number indicating elliptical mirror in R-axis, angle from $R_{00}-\Theta_{00}-Z_{00}$ to $R_{j 0}-\Theta_{j 0}-Z_{j 0}$, number of elliptical mirrors in $\Theta$-axis, angle from $\mathrm{F}_{j 0}$ to $\mathrm{F}_{j 1}$, and coordinates of $\mathrm{F}_{j k}$ in multi-part mirror when $f$ is $199.929 \mathrm{~mm}$.

\begin{tabular}{|c|c|c|c|c|c|c|c|c|c|c|c|c|c|c|}
\hline \multirow[b]{3}{*}{$j$} & \multirow[b]{3}{*}{$\xi_{\mathrm{F} j}\left[^{\circ}\right]$} & \multicolumn{2}{|c|}{$l[\mathrm{~mm}]$} & $s[\mathrm{~mm}]$ & \multicolumn{2}{|c|}{$f[\mathrm{~mm}]$} & \multicolumn{2}{|c|}{$r_{\mathrm{E}}[\mathrm{mm}]$} & $h[\mathrm{~mm}]$ & \multicolumn{2}{|c|}{$g[\mathrm{~mm}]$} & $n_{\mathrm{R}}$ & $n_{\mathrm{M}}$ & \\
\hline & & & 0.000 & 14.100 & & 929 & & 00 & 0.498 & & 00 & 12 & \multicolumn{2}{|l|}{410} \\
\hline & & $n_{\Theta j}$ & $\theta_{\mathrm{F} j}\left[^{\circ}\right]$ & $r_{\mathrm{F} j k}[\mathrm{~m}$ & & $z_{\mathrm{F} j k}[\mathrm{r}$ & $\mathrm{m}]$ & $j$ & $\xi_{\mathrm{Fj}}\left[^{\circ}\right]$ & $n_{\Theta j}$ & $\theta_{\mathrm{F} j}$ & & $r_{\mathrm{F} j k}[\mathrm{~mm}]$ & $z_{\mathrm{F} j k}[\mathrm{~mm}]$ \\
\hline 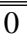 & "0.000 & 1 & 0.000 & 0.0 & & -399 & 005 & 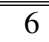 & 3.015 & 37 & 9.7 & & 20.990 & -398.452 \\
\hline 1 & 0.502 & 6 & 59.988 & 3.5 & & -398 & 989 & 7 & 3.518 & 43 & 8.3 & & 24.484 & -398.253 \\
\hline 2 & 1.005 & 12 & 29.994 & 7.0 & & -398 & 943 & 8 & 4.020 & 50 & 7.1 & & 27.977 & -398.023 \\
\hline 3 & 1.507 & 18 & 19.996 & 10.4 & & -398 & & 9 & 4.523 & 56 & 6.4 & & 31.467 & -397.762 \\
\hline 4 & 2.010 & 25 & 14.398 & 13.9 & & -398 & 759 & 10 & 5.025 & 62 & 5.8 & & 34.955 & -397.471 \\
\hline 5 & 2.512 & 31 & 11.608 & 17.4 & & -398 & 621 & 11 & 5.528 & 69 & 5.2 & & 38.440 & -397.149 \\
\hline
\end{tabular}

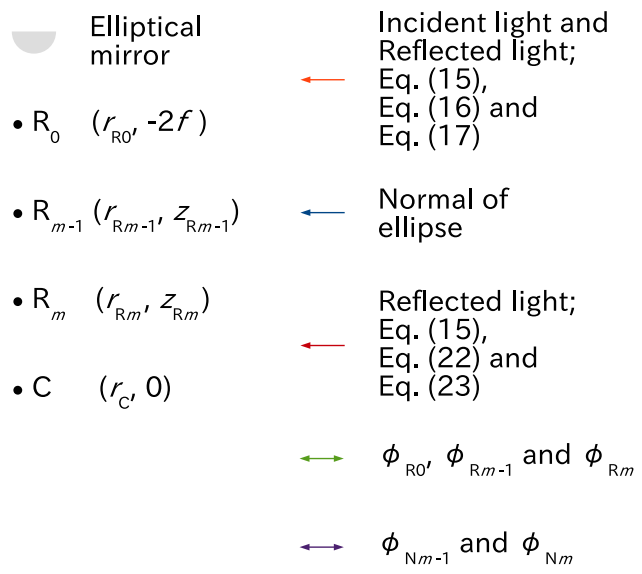

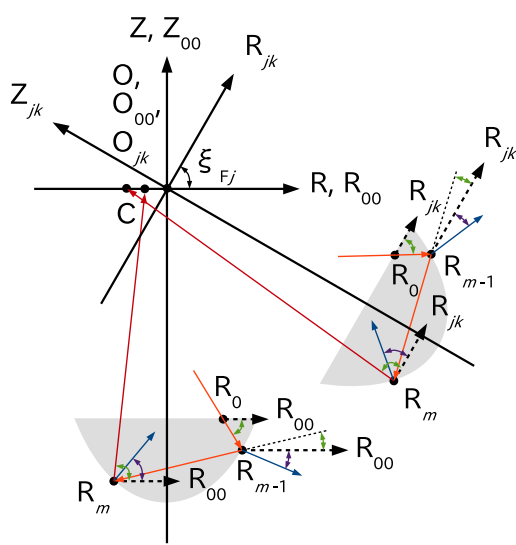

Figure 3: Analysis method of single elliptical and multi-part mirrors.

Using Eqs. (15) and (18), coordinate $z_{\mathrm{R} m}$ can be expressed as

$$
z_{\mathrm{R} m}=a r_{\mathrm{R} m}+b
$$

Using Eqs. (18) and (19), angle $\phi_{\mathrm{N} m}$ can be expressed as

$$
\tan \phi_{\mathrm{N} m}=\frac{s^{2} z_{\mathrm{R} m}}{l^{2} r_{\mathrm{R} m}}
$$

Using Eq. (20), angle $\phi_{\mathrm{R} m}$ can be expressed as

$$
\phi_{\mathrm{R} m}=2 \phi_{\mathrm{N} m}-\phi_{\mathrm{R} m-1}
$$

In the equation describing the light passing through the point of reflection, $\mathrm{R}_{m}$, using Eq. (15), variable a can be expressed as

$$
a=\tan \phi_{\mathrm{R} m}
$$

Variable $b$ can be expressed as

$$
b=z_{\mathrm{R} m}-\tan \phi_{\mathrm{R} m} r_{\mathrm{R} m}
$$

Using Eqs. (15), (22), and (23), the equation describing the light passing through the point of reflection, $\mathrm{R}_{m}$, in the $\mathrm{R}_{j k^{-}}$ $\mathrm{Z}_{j k}$ plane can be expressed as indicated below. Using Eq. (7), the coordinates $(r, z)$ can be expressed as

$$
\left(\begin{array}{c}
r \\
z
\end{array}\right)=\left(\begin{array}{cc}
\cos \xi_{\mathrm{F} j} & -\sin \xi_{\mathrm{F} j} \\
\sin \xi_{\mathrm{F} j} & \cos \xi_{\mathrm{F} j}
\end{array}\right)\left(\begin{array}{c}
r_{00} \\
z_{00}
\end{array}\right)
$$

Using Eqs. (7), (22), and (23), the equation describing the light passing through the point of reflection, $\mathrm{R}_{m}$, in the R-Z plane can be expressed as

$$
z=\frac{\sin \xi_{\mathrm{F} j}+a \cos \xi_{\mathrm{F} j}}{\cos \xi_{\mathrm{F} j}-a \sin \xi_{\mathrm{F} j}} r+\frac{b}{\cos \xi_{\mathrm{F} j}-a \sin \xi_{\mathrm{F} j}}
$$

Using Eq. (25), the coordinate, $r_{\mathrm{C}}$, can be expressed as

$$
r_{\mathrm{C}}=-\frac{b}{\sin \xi_{\mathrm{F} j}+a \cos \xi_{\mathrm{F} j}}
$$

\section{Experimental Method}

4.1 Manufacturing Mirrors Using the design values listed in Table 2, we manufactured actual single elliptical mirrors with a focal length $f$ of $17.503 \mathrm{~mm}$ and a radius $r_{\mathrm{E}}$ of $0.98 \mathrm{~mm}$ and with a focal length $f$ of $17.504 \mathrm{~mm}$ and a radius $r_{\mathrm{E}}$ of $32.697 \mathrm{~mm}$. Fig. 4 shows images of the single elliptical mirror. Fig. 4(a) shows a single elliptical mirror with a focal length $f$ of $17.503 \mathrm{~mm}$ and a radius $r_{\mathrm{E}}$ of $0.98 \mathrm{~mm}$, and Fig. 4(b) shows a single elliptical mirror with a focal length $f$ of $17.504 \mathrm{~mm}$ and a radius $r_{\mathrm{E}}$ of 32.697 $\mathrm{mm}$. For the single elliptical mirror with a focal length $f$ of $17.503 \mathrm{~mm}$ and a radius $r_{\mathrm{E}}$ of $0.98 \mathrm{~mm}$, the substrate is black polycarbonate. Aluminum was vapor-deposited on 
Table 5: Specifications of light receiver, light-shielding sheet, first focal point, and second focal point in measured objects of dome light, light-shielded single elliptical mirror, light-shielded multi-part mirror, single elliptical mirror, and multi-part mirror in experimental set-up.

\begin{tabular}{c|c|c|c|c} 
Object name & Light receiver & Light-shielding sheet & First focal point & Second focal point \\
\hline \hline Dome light & $\mathrm{F}$ & - & - & - \\
\hline Light-shielded single elliptical mirror & $\mathrm{O}$ & $\mathrm{F}$ & $\mathrm{F}$ & $\mathrm{O}$ \\
\hline Light-shielded multi-part mirror & $\mathrm{O}$ & $\mathrm{S}$ & $\mathrm{F}$ & $\mathrm{O}$ \\
\hline Single elliptical and multi-part mirrors & $\mathrm{O}$ & - & $\mathrm{F}$ & $\mathrm{O}$
\end{tabular}

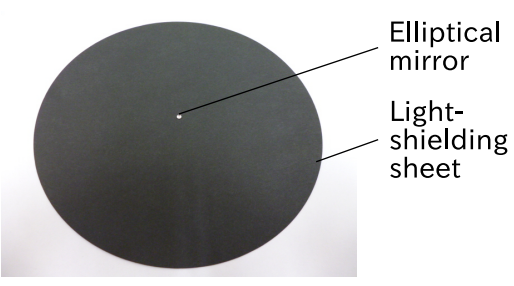

(a) $f$ is $17.503 \mathrm{~mm}$, and $r_{\mathrm{E}}$ is $0.98 \mathrm{~mm}$

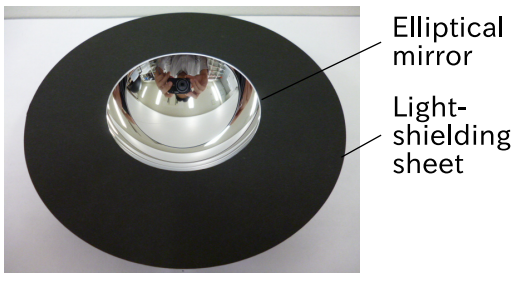

(b) $f$ is $17.504 \mathrm{~mm}$, and $r_{E}$ is $32.697 \mathrm{~mm}$

Figure 4: Images showing elliptical mirror and light-shielding sheet in single elliptical mirrors.

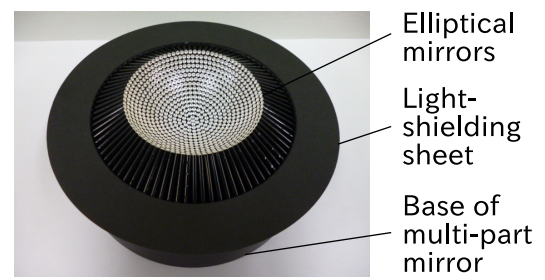

Figure 5: Image showing elliptical mirror, light-shielding sheet, and base in multi-part mirror when $f$ is $17.503 \mathrm{~mm}$.

the mirror surface, after which silicon dioxide was vapordeposited as a protective film. The arithmetic mean roughness of the mirror surface is $1.17 \mu \mathrm{m}$. For the single elliptical mirror with a focal length $f$ of $17.504 \mathrm{~mm}$ and a radius $r_{\mathrm{E}}$ of $32.697 \mathrm{~mm}$, the substrate is heat-resistant glass. Aluminum was vapor-deposited on the mirror surface, after which silicon dioxide was vapor-deposited as a protective film. The reflectivity of the mirror surface is more than $88 \%$ at wavelengths from $400 \mathrm{~nm}$ to $700 \mathrm{~nm}$. The parts other than the mirror surface of this single elliptical mirror were covered with a light-shielding sheet.

Using the design values listed in Table 3, we manufactured an actual multi-part mirror with a focal length $f$ of $17.503 \mathrm{~mm}$, whose images are displayed in Fig. 5. The

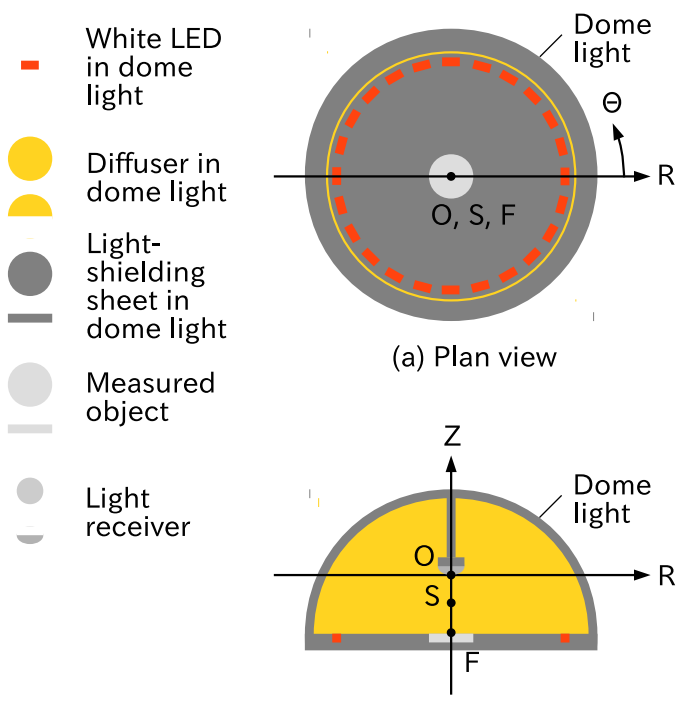

(b) Longitudinal view

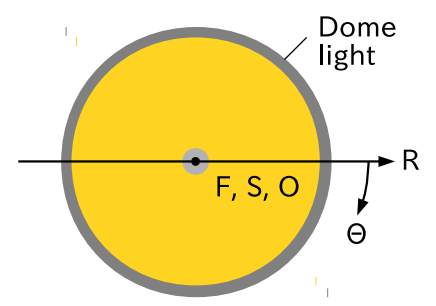

(c) Front view

Figure 6: Mechanism of white LED, diffuser, and light-shielding sheet in dome light; measured object; and light receiver in experimental set-up from (a) plan, (b) longitudinal, and (c) front views.

multi-part mirror is composed of multiple elliptical mirrors and a base. The elliptical mirrors of the multi-part mirror are identical to the manufactured single elliptical mirror with a focal length $f$ of $17.503 \mathrm{~mm}$ and a radius $r_{\mathrm{E}}$ of 0.98 $\mathrm{mm}$. The base of the multi-part mirror is made of an aluminum alloy. The surface of the base was anodized in matte black to prevent light from reflecting off it and subsequently covered with a light-shielding sheet.

4.2 Experimental Set-up Figure 6 shows the structure of the experimental set-up. Figures 6(a)-(c) present the plan, longitudinal, and front views, respectively. The experimental set-up consists of a dome light, measured object, and light receiver. The experimental set-up is covered with a light-shielding sheet to block stray light that could affect 


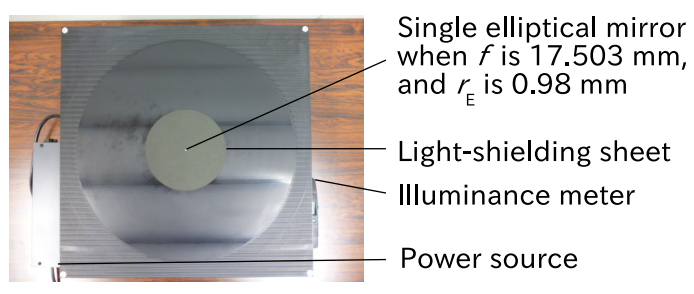

(a) Plan view

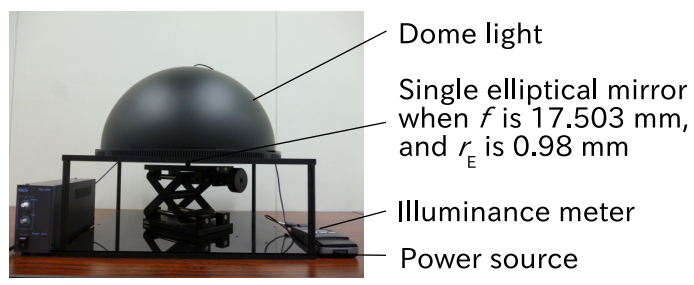

(b) Side view

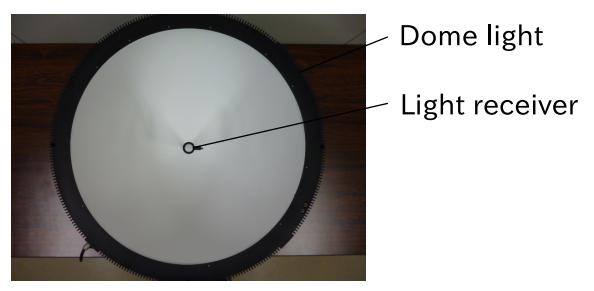

(c) Front view

Figure 7: Images of single elliptical mirror when $f$ is 17.503 $\mathrm{mm}$ and $r_{\mathrm{E}}$ is $0.98 \mathrm{~mm}$, light-shielding sheet, illuminance meter, power source, dome light, and light receiver in the experimental set-up from (a) plan, (b) side, and (c) front views.

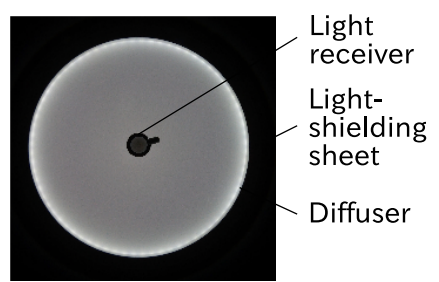

Figure 8: Image of light diffused by diffuser and light-shielding sheet in dome light.

the measurements. An LED light source and a diffuser are installed inside the dome light. The diffuser is in the shape of a half celestial sphere created by rotating a semicircle on the $\mathrm{R}-\mathrm{Z}$ plane in the $\mathrm{R}-\Theta-\mathrm{Z}$ coordinate system around the $\mathrm{Z}$ axis. The equation of the semicircle can be expressed as

$$
r^{2}+(z+2 f)^{2}=40000 \quad(z \geqq-2 f)
$$

The diffuser can generate diffused light inside the dome light. The measured object and the light receiver are positioned with the $\mathrm{Z}$ axis as the central axis. Depending on what is to be measured, the measured object and the light receiver are fixed at positions along the $\mathrm{Z}$ axis.

Table 5 lists the specifications of the measured objects in the experimental set-up. Figure 6 shows the positions of the light-shielding sheet, measured object, and light receiver in the measured object in the experimental set-up. The light receivers are installed at the focal point, $\mathrm{F}$, and the origin, $\mathrm{O}$, and oriented in the positive and negative direction of the $\mathrm{Z}$ axis, respectively. The light-shielding sheet is installed at the focal point, F, for the light-shielded single elliptical mirror and at point $S$ for the light-shielded multi-part mirror. For the light-shielded single elliptical and multi-part mirrors and the single elliptical and multi-part mirrors, the first focal point is set at the focal point, $\mathrm{F}$, and the second focal point is set at the origin, $\mathrm{O}$.

Figure 7 shows the images of the experimental set-up, with Figs. 7(a)-(c) displaying the plan, side, and front views, respectively. In Fig. 7, the measured object is the single elliptical mirror with a focal length $f$ of $17.503 \mathrm{~mm}$ and a radius $r_{\mathrm{E}}$ of $0.98 \mathrm{~mm}$. The dome light is connected to a power source. The light receiver is connected to an illuminance meter. The dome light is model HPD2-400SWCR14, and the power source is model PD2-5024, both manufactured by CCS Inc. HPD2-400SW-CR14 can generate diffused light by illuminating the diffuser inside the dome light from the white LED installed inside the dome light. PD2-5024 allows a dimmer control of the dome light. A total of 16 levels of coarse dimming and 16 levels of fine dimming can be combined to produce 256 levels of dimming. In the experimental set-up, we used coarse dimming to adjust the dimming level and fixed the fine dimming to one level. The light receiver and illuminance meter are model T10MA from Konica Minolta Inc. T-10MA can measure the illuminance at the light receiver and display the measured illuminance on the illuminance meter. The light receiver has a radius of $7 \mathrm{~mm}$, and the light-receiving direction is hemispherical. The range of illuminance that can be measured is from $0.011 x$ to $299,9001 x$.

Figure 8 shows an image of the diffused light on the inside of the dome light. The dimming level of PD2-5024 was set as 2. The image was captured with Kodak SP360. SP360 can shoot at a $\Theta$-axis direction of $360^{\circ}$ and a Z-axis direction of $214^{\circ}$. The lens of SP360 was placed at the focal point, F, and oriented in the positive $\mathrm{Z}$-axis direction. The figure shows the diffused light generated from the diffuser at $360^{\circ}$ in the $\Theta$ direction and $180^{\circ}$ in the $\mathrm{Z}$ direction, except at the light receiver.

4.3 Measurement The illuminance of the dome light, light-shielded measured object, measured object, and measured object only and the illuminance ratio indicating the light-concentrating efficiency are denoted as $i_{\mathrm{D}}, i_{\mathrm{S}}, i_{\mathrm{M}}, i_{\mathrm{T}}$, and $e$, respectively. Illuminance $i_{\mathrm{T}}$ is the value obtained by subtracting illuminance $i_{\mathrm{S}}$ from illuminance $i_{\mathrm{M}}$. The illuminance ratio, $e$, is the ratio of illuminance $i_{\mathrm{T}}$ to illuminance $i_{\mathrm{D}}$. Illuminance $i_{\mathrm{T}}$ can be expressed as

$$
i_{\mathrm{T}}=i_{\mathrm{M}}-i_{\mathrm{S}}
$$

Using Eq. (28), the illuminance ratio, $e$, can be expressed as

$$
e=\frac{i_{\mathrm{T}}}{i_{\mathrm{D}}}
$$


Table 6: Comparison of dimming level with illuminances of dome light, light-shielded measurement object, measured object, and only measured object as well as the illuminance ratio of single elliptical mirrors.

\begin{tabular}{r|r|r|r|r|r|r|r|c}
$f[\mathrm{~mm}]$ & $r_{\mathrm{E}}[\mathrm{mm}]$ & $n_{\mathrm{M}}$ & Dimming level & $i_{\mathrm{D}}[\mathrm{lx}]$ & $i_{\mathrm{S}}[\mathrm{lx}]$ & $i_{\mathrm{M}}[1 \mathrm{x}]$ & $i_{\mathrm{T}}[\mathrm{xx}]$ & $e$ \\
\hline \hline 17.503 & 0.980 & 1 & 2 & $1,031.0$ & 241.3 & 241.6 & 0.3 & 0.0002910 \\
\hline 17.504 & 32.697 & 1 & 2 & $1,031.0$ & 241.3 & 538.0 & 296.7 & 0.2870000 \\
\hline 199.929 & 1.000 & 1 & 2 & $1,172.0$ & - & - & - & 0.0000479
\end{tabular}

We experimentally determined the relationship between the dimming level and illuminance $i_{\mathrm{D}}$ of the dome light. We measured illuminance $i_{\mathrm{D}}$ with the dimming level of the dome light set as 2 . We compared the measured illuminance $i_{\mathrm{S}}$ and $i_{\mathrm{M}}$ of the measured object to each other and to illuminance $i_{\mathrm{T}}$ of the measured object only. Subsequently, we determined illuminance $i_{\mathrm{T}}$ from illuminance $i_{\mathrm{S}}$ and $i_{\mathrm{M}}$. We compared the illuminance ratio, $e$, in the measured object and then determined it from illuminance $i_{\mathrm{T}}$.

\section{Performance Evaluation}

5.1 Analysis Results We analyzed the relationship between angle $\phi_{\mathrm{R} 0}$ and coordinate $r_{\mathrm{C}}$ of the single elliptical and multi-part mirrors. We used Eqs. (15)-(26) for this by calculating the value of coordinate $r_{\mathrm{C}}$ at $1^{\circ}$ increments of angle $\phi_{\mathrm{R} 0}$. The range of angle $\phi_{\mathrm{R} 0}$ was set from $-90^{\circ}$ to $90^{\circ}$ to cover a half celestial sphere of $180^{\circ}$ in the $\mathrm{Z}$-axis direction. Coordinate $r_{\mathrm{C}}$ ranged from $-7 \mathrm{~mm}$ to $7 \mathrm{~mm}$ to cover the radius of the light receiver. We compared the single elliptical mirrors with a focal length $f$ of $17.503 \mathrm{~mm}$ and a radius $r_{\mathrm{E}}$ of $0.98 \mathrm{~mm}$ and with a focal length $f$ of 199.929 $\mathrm{mm}$ and a radius $r_{\mathrm{E}}$ of $1 \mathrm{~mm}$. We also compared the multipart mirrors with focal lengths $f$ of $17.503 \mathrm{~mm}$ and 199.929 $\mathrm{mm}$. The ratio of coordinate $r_{\mathrm{R} 0}$ to radius $r_{\mathrm{E}}$ is denoted as $r_{\mathrm{R} 0} / r_{\mathrm{E}}$. For the single elliptical and multi-part mirrors, we compared the relationship between angle $\phi_{\mathrm{R} 0}$ and coordinate $r_{\mathrm{C}}$ symmetrically about the $\mathrm{Z}$ axis at the following values of $r_{\mathrm{R} 0} / r_{\mathrm{E}}: 0,0.0979,0.5$, and 1 . For the multi-part mirrors, we compared the relationship between angle $\phi_{\mathrm{R} 0}$ and coordinate $r_{\mathrm{C}}$ symmetrically about the $\mathrm{Z}$ axis at the following values of $r_{\mathrm{R} 0} / r_{\mathrm{E}}:-1,-0.5,-0.0979,0,0.0979,0.5$ and 1. In each case, we took measurements at angles $\xi_{\mathrm{F} 0}, \xi_{\mathrm{F} 8}$, and $\xi_{\mathrm{F} 16}$ or alternatively at angles $\xi_{\mathrm{F} 0}$ and $\xi_{\mathrm{F} 11}$. We plotted all the relationships between angle $\phi_{\mathrm{R} 0}$ and coordinate $r_{\mathrm{C}}$ at $r_{\mathrm{R} 0} / r_{\mathrm{E}}$ values of -0.0979 and 0.0979 for the single elliptical mirror with a focal length $f$ of $17.503 \mathrm{~mm}$ and a radius $r_{\mathrm{E}}$ of $0.98 \mathrm{~mm}$ and the multi-part mirror with a focal length $f$ of $17.503 \mathrm{~mm}$ at angle $\xi_{\mathrm{F} 0}$.

Figures 9-12 compare the relationships for the single elliptical mirrors, and Figs. 13-16 compare them for the multi-part mirror with a focal length $f$ of $17.503 \mathrm{~mm}$ at angles $\xi_{\mathrm{F} 8}$, and $\xi_{\mathrm{F} 16}$. Figures 17-20 compare the relationships for the multi-part mirror with focal lengths $f$ of $17.503 \mathrm{~mm}$ and $199.929 \mathrm{~mm}$. In these figures when the ratio of $r_{\mathrm{R} 0} / r_{\mathrm{E}}$ is greater than the range from 0 to 1 or smaller than the range from 0 to -1 , less of the light incident from $-90^{\circ}$ to $90^{\circ}$, which is the range of angle $\phi_{\mathrm{R} 0}$, is concentrated from $-7 \mathrm{~mm}$ to $7 \mathrm{~mm}$, which is the range of coordinate $r_{\mathrm{C}}$. When the focal length, $f$, and the radius, $r_{\mathrm{E}}$, of the single elliptical mirrors are increased, less light is concentrated at each

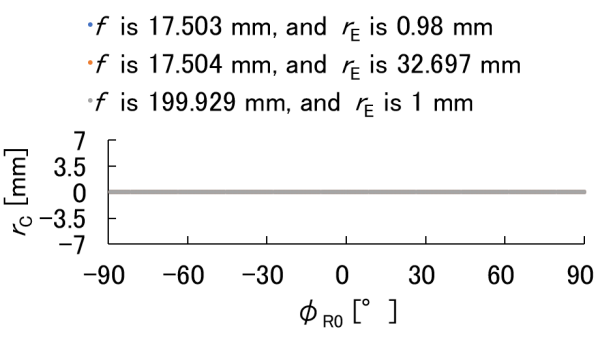

Figure 9: Comparison of relationship of angle between incident light at $\mathrm{R}_{0}$ and $\mathrm{R}_{j k}$ and coordinate of $\mathrm{C}$ when $r_{\mathrm{R} 0} / r_{\mathrm{E}}$ is 0 in single elliptical mirrors.

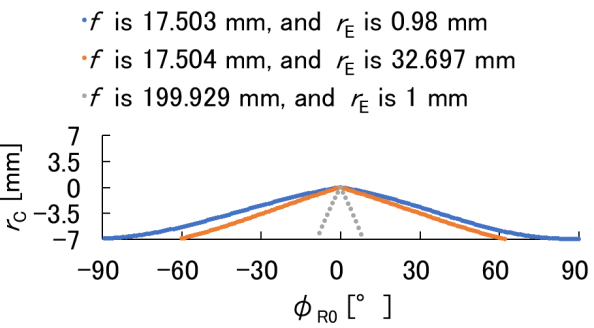

Figure 10: Comparison of relationship of angle between incident light at $\mathrm{R}_{0}$ and $\mathrm{R}_{j k}$ and coordinate of $\mathrm{C}$ when $r_{\mathrm{R} 0} / r_{\mathrm{E}}$ is 0.0979 in single elliptical mirrors.

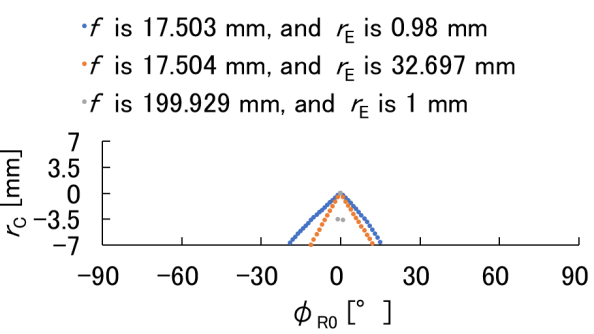

Figure 11: Comparison of relationship of angle between incident light at $\mathrm{R}_{0}$ and $\mathrm{R}_{j k}$ and coordinate of $\mathrm{C}$ when $r_{\mathrm{R} 0} / r_{\mathrm{E}}$ is 0.5 in single elliptical mirrors.

ratio $r_{\mathrm{R} 0} / r_{\mathrm{E}}$. In the case of the multi-part mirrors, less light is concentrated as the angle, $\xi_{\mathrm{F} j}$, and the focal length, $f$, increase.

5.2 Experiment Results Figure 21 shows the appearance of the measured objects inside the experimental set-up during the experiment. Figures 21(a)-(c) present the dome light, light-shielded single elliptical mirrors with a focal length $f$ of $17.503 \mathrm{~mm}$ and a radius $r_{\mathrm{E}}$ of $0.98 \mathrm{~mm}$ and with a focal length $f$ of $17.504 \mathrm{~mm}$ and a radius $r_{\mathrm{E}}$ of 32.697 $\mathrm{mm}$, and (c) light-shielded multi-part mirror with a focal 


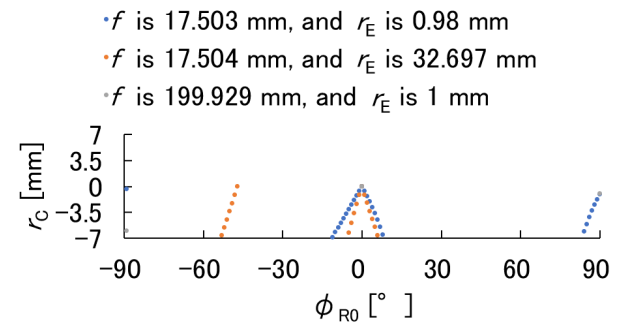

Figure 12: Comparison of relationship of angle between incident light at $\mathrm{R}_{0}$ and $\mathrm{R}_{j k}$ and coordinate of $\mathrm{C}$ when $r_{\mathrm{R} 0} / r_{\mathrm{E}}$ is 1 in single elliptical mirrors.

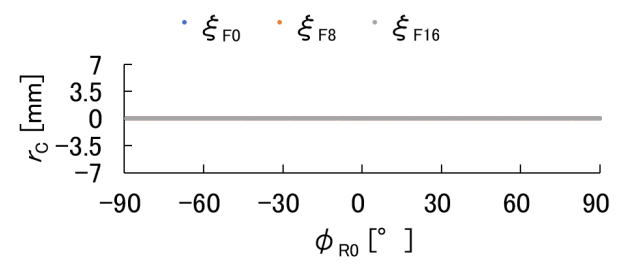

Figure 13: Comparison of relationship of angle between incident light at $\mathrm{R}_{0}$ and $\mathrm{R}_{j k}$ and coordinate of $\mathrm{C}$ when $r_{\mathrm{R} 0} / r_{\mathrm{E}}$ is 0 at $\xi_{\mathrm{F} 0}, \xi_{\mathrm{F} 8}$, and $\xi_{\mathrm{F} 16}$ in multi-part mirror when $f$ is 17.503 $\mathrm{mm}$.

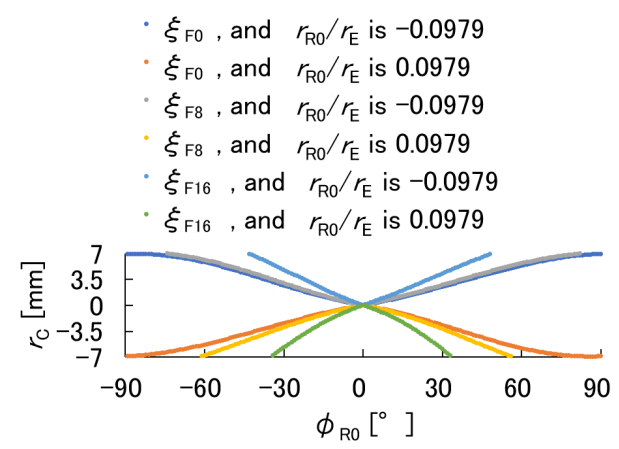

Figure 14: Comparison of relationship of angle between incident light at $\mathrm{R}_{0}$ and $\mathrm{R}_{j k}$ and coordinate of $\mathrm{C}$ when $r_{\mathrm{R} 0} / r_{\mathrm{E}}$ is -0.0979 and 0.0979 at $\xi_{\mathrm{F} 0}, \xi_{\mathrm{F} 8}$, and $\xi_{\mathrm{F} 16}$ in multi-part mirror when $f$ is $17.503 \mathrm{~mm}$.

length $f$ of $17.503 \mathrm{~mm}$, respectively. Figures 21(d)-(f) show the single elliptical mirror with a focal length $f$ of 17.503 $\mathrm{mm}$ and radius $r_{\mathrm{E}}$ of $0.98 \mathrm{~mm}$, single elliptical mirror with a focal length $f$ of $17.504 \mathrm{~mm}$ and radius $r_{\mathrm{E}}$ of $32.697 \mathrm{~mm}$, and multi-part mirror with a focal length $f$ of $17.503 \mathrm{~mm}$, respectively. This is the view from the negative $\mathrm{Z}$-axis direction with the dimming level set as 2 . The images of the dome light and the light-shielded single elliptical and multipart mirrors demonstrate the light-shielding sheet reflecting the diffused light. Therefore, it is necessary to determine illuminance $i_{\mathrm{T}}$, which is the difference between illuminance $i_{\mathrm{M}}$ and $i_{\mathrm{S}}$. The images of the single elliptical and multipart mirrors show that the mirrors can brightly reflect the diffused light.

Table 6 lists the dome light dimming level, illuminance $i_{\mathrm{D}}, i_{\mathrm{S}}, i_{\mathrm{M}}$, and $i_{\mathrm{T}}$ values, and illuminance ratio $e$ for the sin-

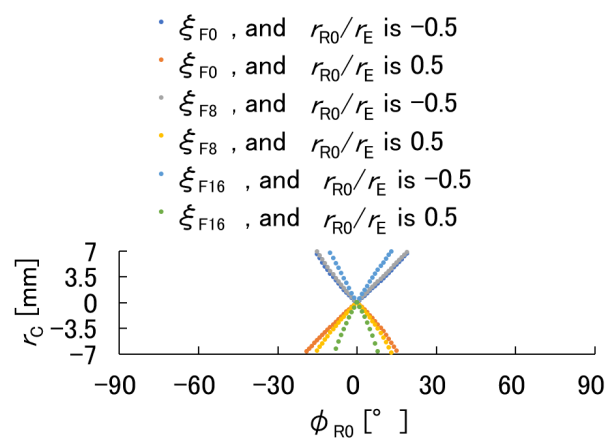

Figure 15: Comparison of relationship of angle between incident light at $\mathrm{R}_{0}$ and $\mathrm{R}_{j k}$ and coordinate of $\mathrm{C}$ when $r_{\mathrm{R} 0} / r_{\mathrm{E}}$ is -0.5 and 0.5 at $\xi_{\mathrm{F} 0}, \xi_{\mathrm{F} 8}$, and $\xi_{\mathrm{F} 16}$ in multi-part mirror when $f$ is $17.503 \mathrm{~mm}$.

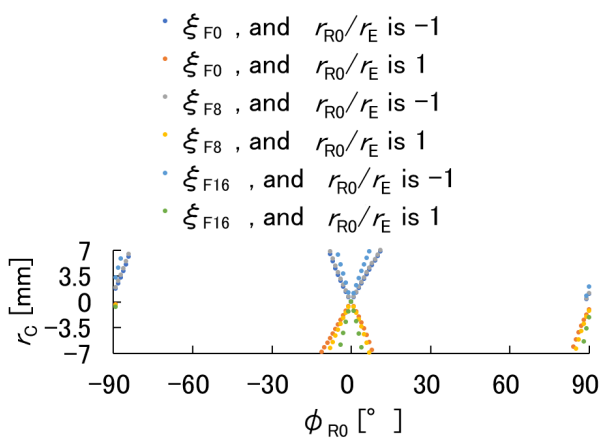

Figure 16: Comparison of relationship of angle between incident light at $\mathrm{R}_{0}$ and $\mathrm{R}_{j k}$ and coordinate of $\mathrm{C}$ when $r_{\mathrm{R} 0} / r_{\mathrm{E}}$ is -1 and 1 at $\xi_{\mathrm{F} 0}, \xi_{\mathrm{F} 8}$, and $\xi_{\mathrm{F} 16}$ in multi-part mirror when $f$ is $17.503 \mathrm{~mm}$

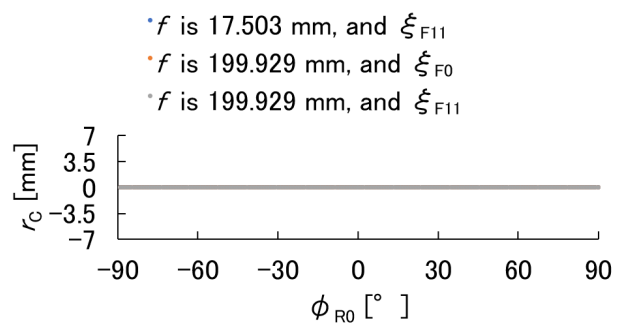

Figure 17: Comparison of relationship of angle between incident light at $\mathrm{R}_{0}$ and $\mathrm{R}_{j k}$ and coordinate of $\mathrm{C}$ when $r_{\mathrm{R} 0} / r_{\mathrm{E}}$ is 0 at $\xi_{\mathrm{F} 0}$ and $\xi_{\mathrm{F} 11}$ in multi-part mirrors.

gle elliptical mirrors. Illuminance $i_{\mathrm{M}}$ is greater than illuminance $i_{\mathrm{S}}$. Therefore, it is necessary to obtain illuminance $i_{\mathrm{T}}$, which is the difference between illuminance $i_{\mathrm{M}}$ and illuminance $i_{\mathrm{S}}$. The illuminance ratio, $e$, for the single elliptical mirror with a focal length $f$ of $199.929 \mathrm{~mm}$ and radius $r_{\mathrm{E}}$ of $1 \mathrm{~mm}$ was estimated based on previous results of Matsumoto [19]. The relationship between the number of mirrors, $n_{\mathrm{M}}$, and the illuminance ratio, $e$, can be expressed as

$$
e=-0.000000004741 n_{\mathrm{M}}{ }^{2}+0.00001138 n_{\mathrm{M}}+0.00003655
$$




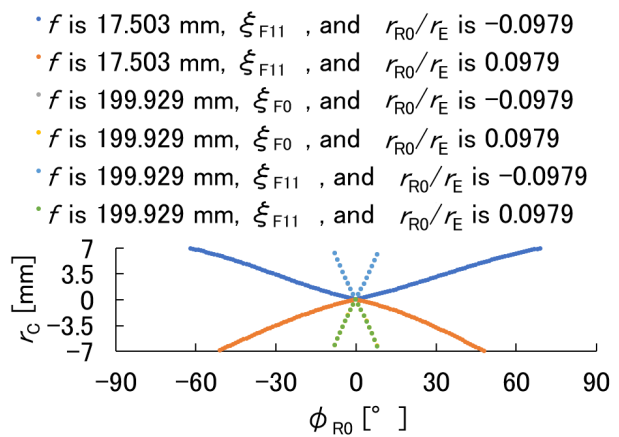

Figure 18: Comparison of relationship of angle between incident light at $\mathrm{R}_{0}$ and $\mathrm{R}_{j k}$ and coordinate of $\mathrm{C}$ when $r_{\mathrm{R} 0} / r_{\mathrm{E}}$ is -0.0979 and 0.0979 at $\xi_{\mathrm{F} 0}$ and $\xi_{\mathrm{F} 11}$ in multi-part mirrors.

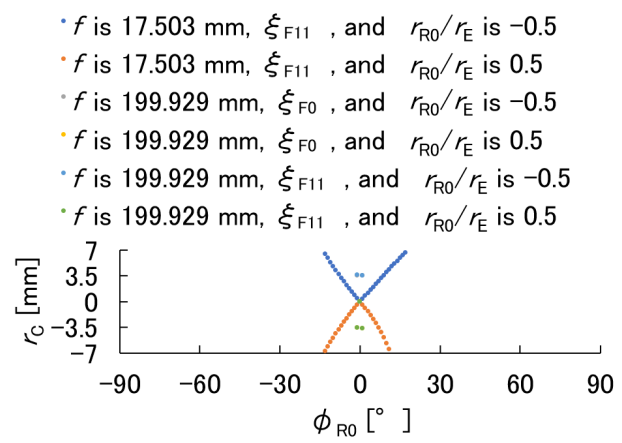

Figure 19: Comparison of relationship of angle between incidentlight at $\mathrm{R}_{0}$ and $\mathrm{R}_{j k}$ and coordinate of $\mathrm{C}$ when $r_{\mathrm{R} 0} / r_{\mathrm{E}}$ is -0.5 and 0.5 at $\xi_{\mathrm{F} 0}$ and $\xi_{\mathrm{F} 11}$ in multi-part mirrors.

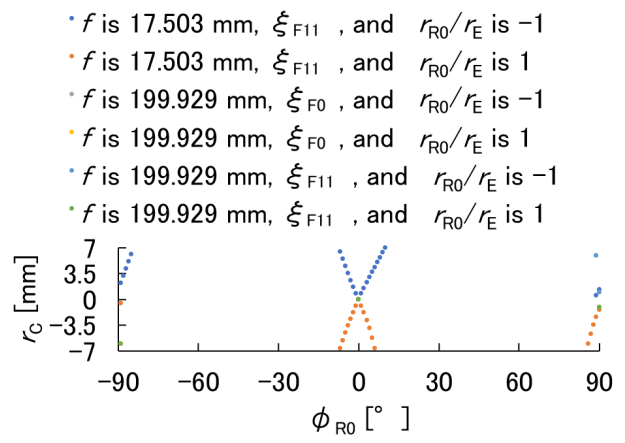

Figure 20: Comparison of relationship of angle between incident light at $\mathrm{R}_{0}$ and $\mathrm{R}_{j k}$ and coordinate of $\mathrm{C}$ when $r_{\mathrm{R} 0} / r_{\mathrm{E}}$ is -1 and 1 at $\xi_{\mathrm{F} 0}$ and $\xi_{\mathrm{F} 11}$ in multi-part mirrors.

Using Eq. (30), the illuminance ratio, $e$, when $n_{\mathrm{M}}$ is 1 is estimated as 0.0000479 . Because illuminance $i_{\mathrm{D}}$ is different for the three single elliptical mirrors, we compared their values of the illuminance ratio, $e$. First, we compared the single elliptical mirror with a focal length $f$ of $17.503 \mathrm{~mm}$ and a radius $r_{\mathrm{E}}$ of $0.98 \mathrm{~mm}$ to that with a focal length $f$ of $17.504 \mathrm{~mm}$ and a radius $r_{\mathrm{E}}$ of $32.697 \mathrm{~mm}$. As the radius, $r_{\mathrm{E}}$, increases, the illuminance ratio, $e$, also increases. When the radius, $r_{\mathrm{E}}$, is 33.3 times as large, the illuminance ratio, $e$, is 986 times larger. Next, we compared the single elliptical

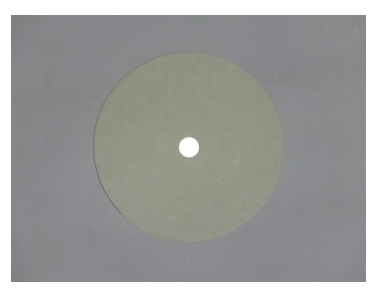

(a) Dome light

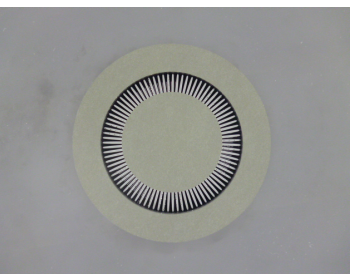

(c) Light-shielded multi-part mirror when $f$ is $17.503 \mathrm{~mm}$

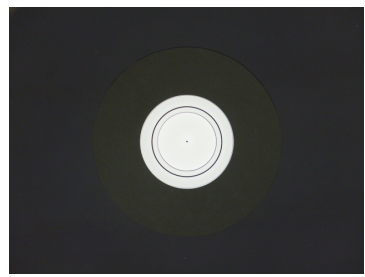

(e) Single elliptical mirror when $f$ is $17.504 \mathrm{~mm}$, and $r_{\mathrm{E}}$ is $32.697 \mathrm{~mm}$

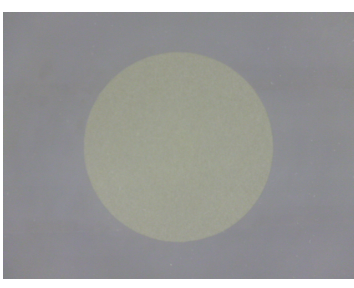

(b) Light-shielded single elliptical mirror when $f$ is $17.503 \mathrm{~mm}$ and $17.504 \mathrm{~mm}$, and $r_{E}$ is $0.98 \mathrm{~mm}$ and $32.697 \mathrm{~mm}$

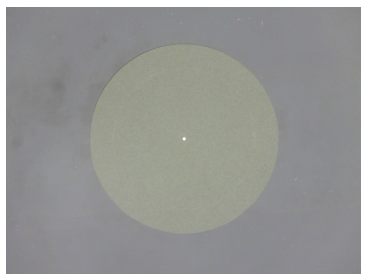

(d) Single elliptical mirror when $f$ is $17.503 \mathrm{~mm}$ and $r_{\mathrm{E}}$ is $0.98 \mathrm{~mm}$

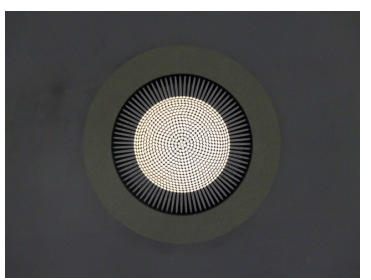

(f) Multi-part mirror when $f$ is $17.503 \mathrm{~mm}$
Figure 21: Images of measured objects with dome light, light-shielded single elliptical and multi-part mirrors, single elliptical and multi-part mirrors in experimental set-up.

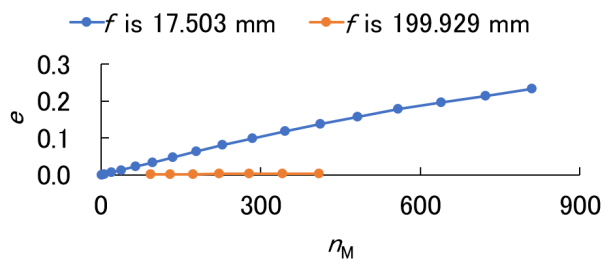

Figure 22: Comparison of relationship between number of all elliptical mirrors and illuminance ratio in multi-part mirrors.

mirror with a focal length $f$ of $17.503 \mathrm{~mm}$ and a radius $r_{\mathrm{E}}$ of $0.98 \mathrm{~mm}$ to that with a focal length $f$ of $199.929 \mathrm{~mm}$ and a radius $r_{\mathrm{E}}$ of $1 \mathrm{~mm}$. As the focal length $f$ decreases, the illuminance ratio, $e$, increases. When the focal length $f$ is 0.0875 times as large, the illuminance ratio, $e$, is 6.07 times larger. This shows that to increase the illuminance ratio, $e$, it is more effective to change the radius, $r_{\mathrm{E}}$, than modify the focal length, $f$. 
Figure 22 compares the relationship between the number of mirrors, $n_{\mathrm{M}}$, and the illuminance ratio, $e$, for the multipart mirrors. We also compared the multi-part mirrors with focal lengths $f$ of $17.503 \mathrm{~mm}$ and $199.929 \mathrm{~mm}$. For the multi-part mirror with a focal length $f$ of $199.929 \mathrm{~mm}$, the relationship between the number of mirrors, $n_{\mathrm{M}}$, and the illuminance ratio, $e$, is the result obtained by Matsumoto [19]. As the number of mirrors, $n_{\mathrm{M}}$, increases, the illuminance ratio, $e$, increases. The rate of increase in the illuminance ratio, $e$, is greater for the multi-part mirror with a focal length $f$ of $17.503 \mathrm{~mm}$ than that for the multi-part mirror with a focal length $f$ of $199.929 \mathrm{~mm}$. Furthermore, the increase in the illuminance ratio, $e$, is nonlinear. As the number of mirrors, $n_{\mathrm{M}}$, increases, the rate of increase in the illuminance ratio, $e$, decreases. The analysis results in Figs. 13-20 show that as the angle, $\xi_{\mathrm{F} j}$, and the focal length, $f$, increase, less light is concentrated. To solve this problem, it is necessary for the multi-part mirror to possess a three-dimensional collector instead of a flat collector surface to accommodate a large angle $\xi_{\mathrm{F} j}$.

\section{Conclusion}

In this study, we installed a collector surface on the second focal point of an elliptical mirror to increase its lightconcentrating efficiency. Subsequently we evaluated single elliptical and multi-part mirrors that capture diffused light from a half celestial sphere not incident on the first focal point and concentrate it on the collector surface as well as the second focal point. The results obtained in this study are as follows:

1. We configured a coordinate system to design single elliptical and multi-part mirrors and subsequently analyzed the mechanism by which the light would be incident on the designed single elliptical and multi-part mirrors and concentrate on their collector surfaces. Based on the design values, we manufactured actual single elliptical and multi-part mirrors, which were illuminated using our experimental set-up with diffused light and measured the illuminance of the light concentrated on their collector surfaces.

2. We analyzed how the light concentrated on the collector surfaces of the actual single elliptical and multi-part mirrors. As we increased the focal length and the radius of the single elliptical mirrors, we found less light concentrated at each ratio of the coordinate of the point of incidence to the radius. In the case of the multi-part mirrors, less light was concentrated as we increased the angles and the focal lengths of the elliptical mirrors.

3. We compared the illuminance ratio, which is a measure of the light-concentrating performance, of the single elliptical and multi-part mirrors. For the single elliptical mirrors, as the radius of the elliptical mirror increases, the illuminance ratio also increases. When the radius is 33.3 times as large, the illuminance ratio is 986 times larger. As the focal length of the elliptical mirror decreases, the illuminance ratio increases.
When the focal length is 0.0875 times as large, the illuminance ratio is 6.07 times larger. Therefore, to increase the illuminance ratio, it is more effective to change the radius instead of the focal length. For the multi-part mirrors, we found that increasing the number of elliptical mirrors increases the illuminance ratio. As the focal length decreases, the illuminance ratio increases. As the number of mirrors increases, the illuminance ratio increases. The results of our analysis suggest that as the number of mirrors increases, the angles of the elliptical mirrors become larger, thereby decreasing the amount of concentrated light and slowing the rate of increase in the illuminance ratio. To solve this problem, it is necessary to install a threedimensional collector in a multi-part mirror instead of a flat collector surface to accommodate a larger angle.

\section{Acknowledgements}

This study was funded by the Kurata Grant awarded by the Hitachi Global Foundation, Yashima Environment Technology Foundation, and Takahashi Industrial and Economic Research Foundation. The author expresses his appreciation for the same.

\section{References}

[1] Japan Solar Energy Society ed., "New Solar Energy Utilization Handbook", Japan Solar Energy Society, pp.123-128, Tokyo, 2015 (in Japanese).

[2] Japan Solar Energy Society ed., "Solar Energy Utilization Technology”, Japan Solar Energy Society, p.66, Tokyo, 2006 (in Japanese).

[3] Geospatial Information Authority of Japan ed., "National Atlas of Japan”, Japan Map Center, p.47, Tokyo, 1990 (in Japanese).

[4] R. Winston, "Principles of Solar Concentrators of a Novel Design”, Solar Energy, Vol.16, No.2, pp.89-95, 1974.

[5] G. H. Derrick, I. M. Bassett and D. R. Mills, "Comparison of Reflector Designs for Stationary Tubular Solar Collectors", Solar Energy, Vol.37, No.3, pp.195-203, 1986.

[6] S. S. Rao, H. Lee and Y. Hu, "Optimal Design of Compound Parabolic Concentrator Solar Collector System”, Transactions of the ASME, Journal of Mechanical Design, Vol.136, No.9, pp.091402-1-091402-10, 2014.

[7] G. R. Mather and D. C. Beekley, "Performance of an Evacuated Tubular Collector Using Non-imaging Reflectors", Proceedings of the Joint Conference International Solar Energy Society, No.2, pp.64-78, 1976.

[8] D. R. Mills and J. E. Giutronich, "Symmetrical and Asymmetrical Ideal Cylindrical Radiation Transformers and Concentrators", Journal of the Optical Society of America, Vol.69, No.2, pp.325-328, 1979.

[9] I. M. Bassett and G. H. Derrick, "Diffuse Reflectors in Nonimaging Optics", Proceedings of the International Solar Energy Society Silver Jubilee Congress, Vol.1, pp.557-560, 1979.

IIAE Journal, Vol.9, No.4, 2021 
[10] D. P. Grimmer, "A Comparison of Compound Parabolic and Simple Parabolic Concentrating Solar Collectors", Solar Energy, Vol.22, No.1, pp.21-25, 1979.

[11] J. R. Frissora and D. M. Platt, "Drainable Evacuated Collector with Compound Parabolic Cusp Reflector", Solar Energy and Conservation, Vol.1, pp.113-140, 1980.

[12] B. Norton, P. C. Eames and Y. P. Yadav, "Symmetric and Asymmetric Linear Compound Parabolic Concentrating Solar Energy Collectors - The State-of-the-art in Optical and Thermo-physical Analysis -", International Journal of Ambient Energy, Vol.12, No.4, pp.171-190, 1991.

[13] P. C. Eames and B. Norton, "The Effect of Sky Conditions on the Partition of Incident Solar Energy between the Components of a CPC Solar Energy Collector", Proceedings of the Solar World Congress 1991 (SWC1991), Vol.2, pp.18841889, 1992.

[14] A. Suzuki, "Optimum Design for Non-imaging Solar Concentrators with Reference to Scattering Insolation Fraction", Proceedings of the Japan Solar Energy Society Japan Wind Energy Association Joint Conference, pp.37-40, 1993 (in Japanese).

[15] P. C. Eames, B. Norton, Y. Tripagnostopoulos and P. Yianoulis, "Modelling Line-axis Solar Concentrators in the Medium Temperature Range", Renewable Energy, Vol.16, No.1, pp.743-748, 1999.

[16] J. Wang, L. Yu, C. Jiang, S. Yang and T. Liu, "Optical Analysis of Solar Collector with New V-shaped CPC", Solar Energy, Vol.135, pp.780-785, 2016.

[17] N. Yamada and Y. Honda, "Optical Analysis of High Concentrating Optical Geometry Coupled with Diffuse Solar Radiation Absorber", Journal of Japan Solar Energy Society, Vol.36, No.3, pp.45-50, 2010 (in Japanese).

[18] N. Sellami, T. K. Mallick and D. A. Mcneil, "Optical Characterisation of 3-D Static Solar Concentrator", Energy Conversion and Management, Vol.64, pp.579-586, 2012.

[19] M. Matsumoto, "Multi-part Mirror for Focusing Diffuse Light”, Mechanical Engineering Journal, Vol.4, No.3, 2017, DOI:10.1299/mej.16-00520.

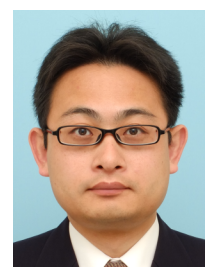

M. Matsumoto (Member) received his Ph.D. degree from University of Tsukuba. He is currently an associate professor at Kanagawa University. His current research interest is mechanical engineering. He is a member of IIAE and so on. 